\title{
Pulsar science with the SKA
}

\author{
Roy Smits ${ }^{1}$, Michael Kramer ${ }^{1}$, Ben Stappers ${ }^{1}$ \\ ${ }^{1}$ Jodrell Bank Centre for Astrophysics, University of Manchester \\ E-mail: Roy.Smits@manchester.ac.uk
}

\begin{abstract}
With its unmatched collective area, the SKA will be capable of detecting the vast majority of pulsars and millisecond pulsars in our Galaxy that are beamed towards the Earth. These pulsars can be used as a detector of gravitational waves and to test General Relativity. Based on the characteristics of the currently known pulsars, we have modeled the pulsar distribution and their detectability throughout the Galaxy. We then studied the effects of different SKA configurations and limitations on computational resources on the speed and sensitivity of an SKA pulsar survey.
\end{abstract}

From planets to dark energy: the modern radio universe

October 1-5 2007

University of Manchester, Manchester, UK 


\section{Introduction}

Pulsars are...

- Neutron stars with a diameter of 20 kilometres.

- Highly magnetized.

- Like lighthouses (emit in a rotating beam), see Fig. 1.

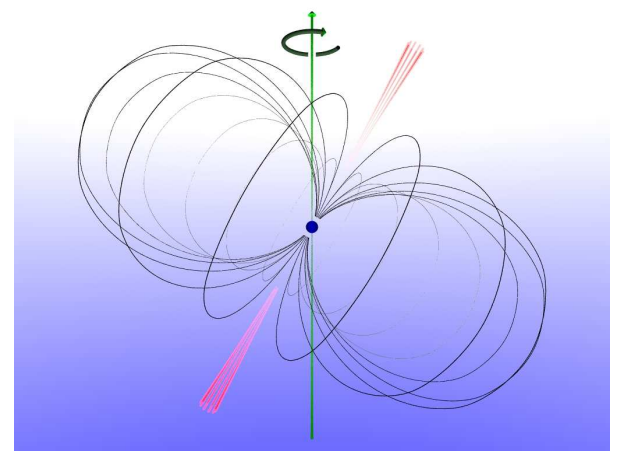

Figure 1: Schematic view of a pulsar. The sphere in the middle represents the neutron star, the curves indicate the magnetic field lines and the protruding cones represent the emission beams.

And they are interesting because...

- A PSR-BH binary will test the limits of General Relativity.

- A pulsar in the galactic center will probe conditions near the $3 \times 10^{6} \mathrm{M}_{\odot}$ black hole.

- (Millisecond) pulsars can be used as gravitational wave detector.

- Understanding pulsar physics.

We therefore want to use the Square Kilometer Array (SKA) to survey the entire sky for normal pulsars, millisecond pulsars and binaries.

\section{Performing the Survey}

To find out how the SKA pulsar survey performance depends on different SKA configurations, we simulated SKA pulsar surveys for different collective areas and different center frequencies. The simulations were performed following Lorimer et al. (2006). The results are shown in Fig. 2. When using the 1-km core of the SKA, a combined survey with the dishes and the Aperture Array (AA) would find about 15000 pulsars.

\section{Limitations}

A pulsar survey with the SKA is hampered by the following.

\section{Beamforming}

A pulsar survey requires realtime calculation of high time resolution 'pencil beams'. Fig. 3 shows the required computation power for different SKA configurations as a function of the size of the core that is used for the beamforming. It also shows how much can be saved on computation power, if the beamforming of the dishes takes place in two stages. This, however, requires the dishes to be placed in identical sub-arrays.

\section{Data rate}

All the data from the 'pencil beams' that are formed need to be transported to either the memory 

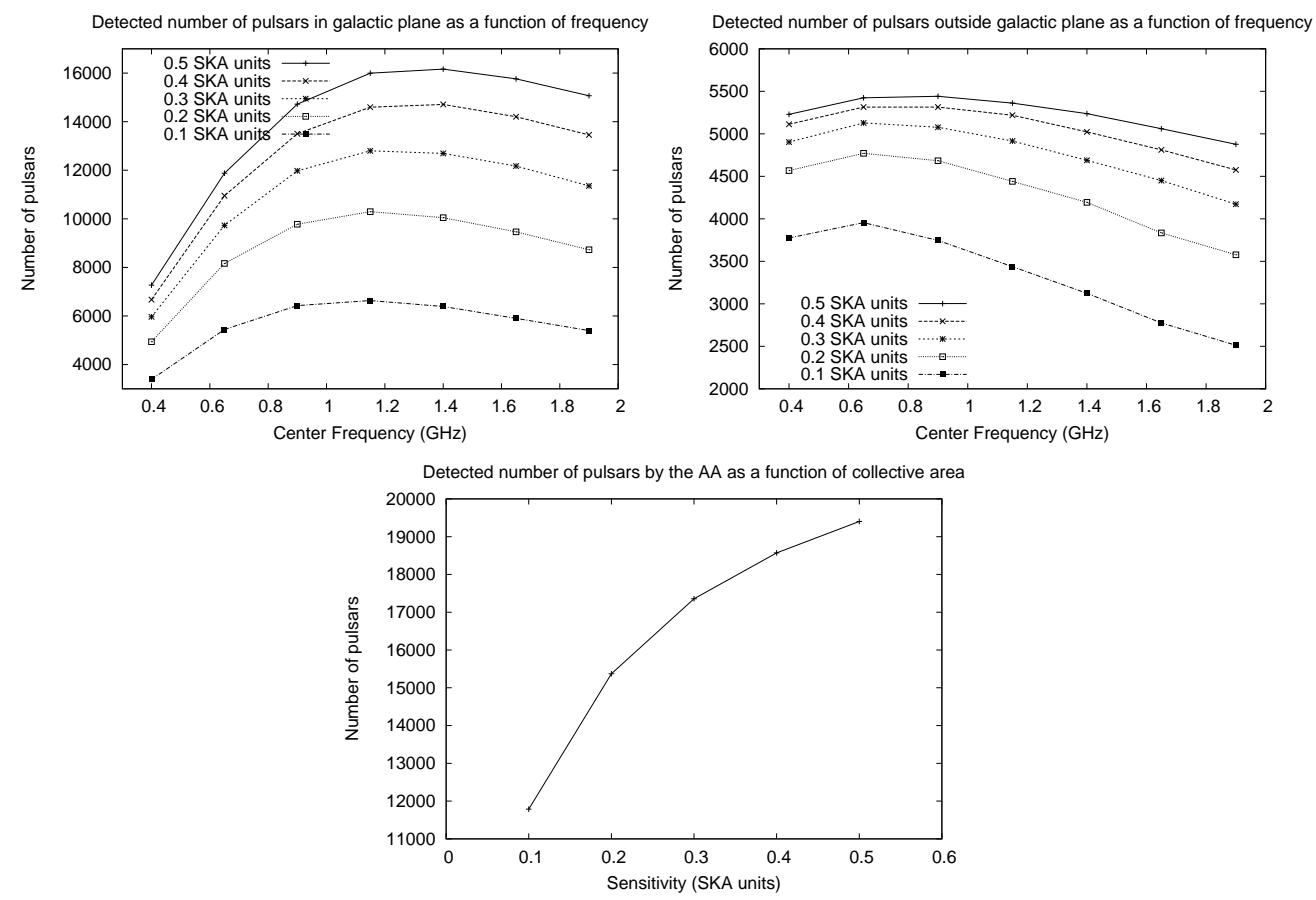

Figure 2: Number of detected pulsars from pulsar survey simulations as a function of centre frequency and for different collecting areas of the SKA. The top left graph shows the number of detected pulsars inside the Galactic plane. The top right graph shows the number of detected pulsars outside the Galactic plane. The bottom graph shows the number of detected pulsars for an all-sky survey using the AA as a function of collecting area.

of a computer or written to a storage device. Fig. 4 shows the amount of data that needs to be transported for different SKA configurations as a function of core-diameter.

\section{Data analysis}

Finally, all the data needs to be searched for accelerated periodic sources. Assuming $N_{D M}$ trial values for the DM and $N_{a c c}$ trial values for the acceleration, then the total number of operations can be estimated as:

$$
N_{\text {oa }}=N_{D M} N_{\text {acc }} \cdot 5 N_{\text {samp }} \log _{2}\left(N_{\text {samp }}\right)
$$

Fig. 5 shows the required computation time to analyse the data realtime for different configurations of the SKA as a function of core-diameter. From these numbers we estimate that only the $1-\mathrm{km}$ core, containing $20 \%$ of the SKA, can be used for a pulsar survey.

\section{Survey Speed}

Assuming $3 \mathrm{deg}^{2}$ FoV and 30 minutes pointing, the survey observation will take 200 days. However, it is likely that the data analysis will take longer. Because of the nature of the search algorithms, it is very expensive to trade collective area for survey speed! 


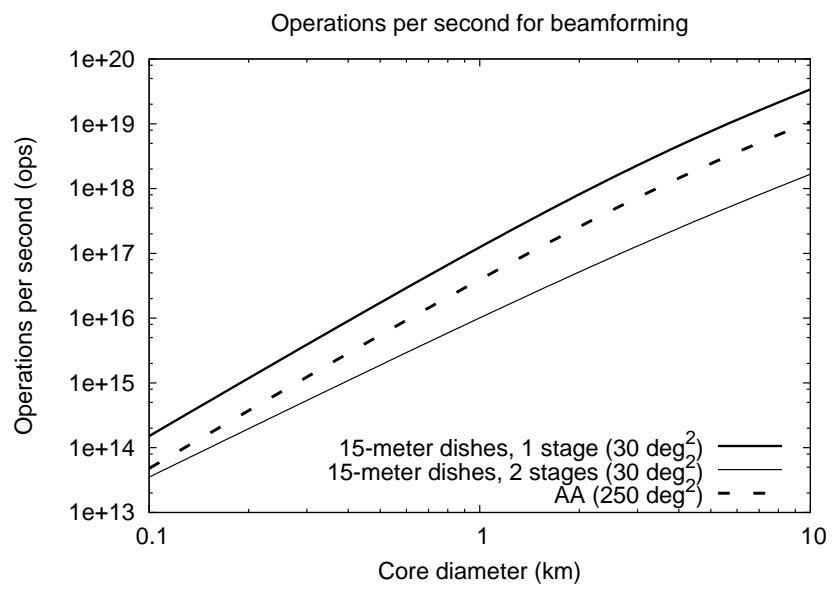

Figure 3: The number of operations per second required to perform the beam forming for 15-m dishes and the AA. It is assumed that there are $240015-\mathrm{m}$ dishes and the bandwidth is $500 \mathrm{MHz}$. For the AA it is assumed that the total collecting area is $500000 \mathrm{~m}^{2}$ and the frequency range is 0.5 to $0.8 \mathrm{GHz}$. In all cases the number of polarisations is 2 .

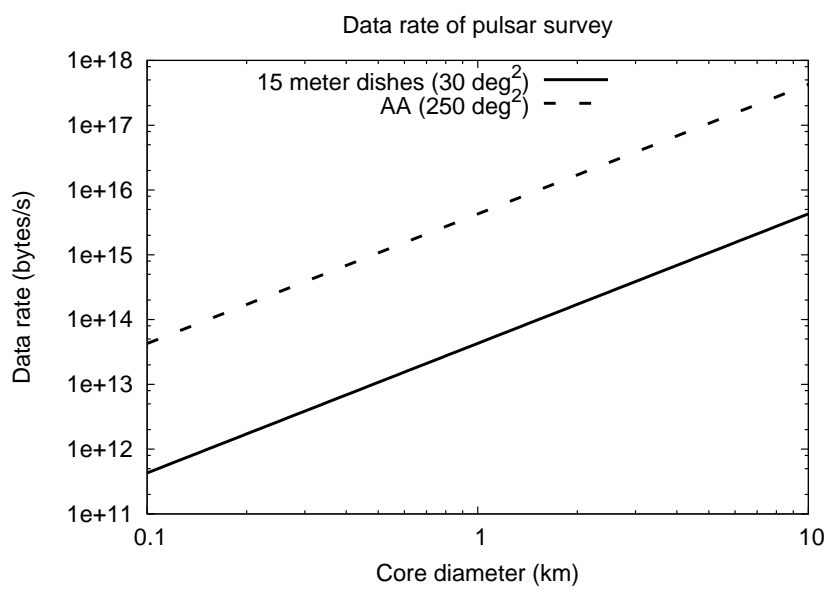

Figure 4: Data rate from pulsar surveys using the 15-m dishes or the AA, as a function of core diameter.

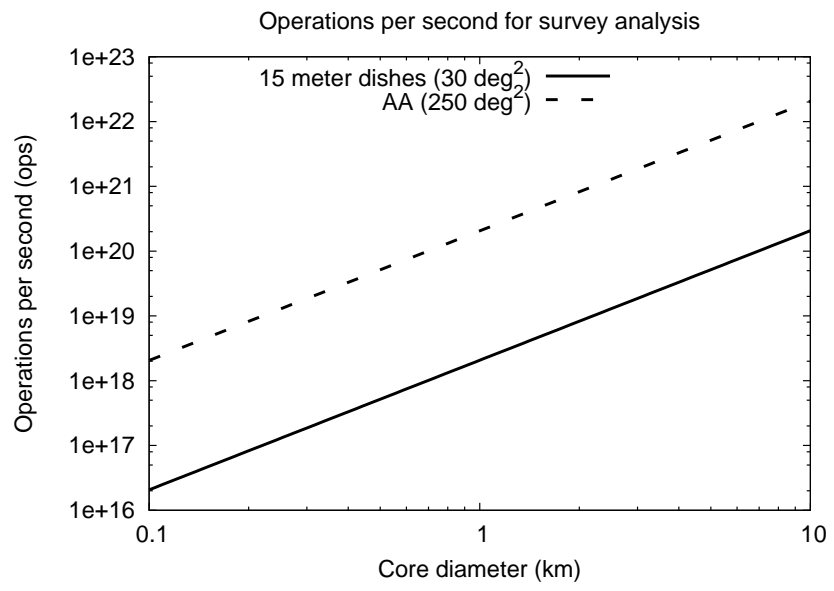

Figure 5: Operations per second required to perform a realtime analysis of pulsar surveys using the 15-m dishes or the AA, as a function of core diameter. 


\section{Timing}

To find interesting pulsars, all newly discoved pulsars need to be timed to a SNR of about 20 once every two weeks for about 6 months. We suggest a simple scheme to optimize the timing of pulsars found by the SKA. Fig. 6 shows the pointing that resulted from applying the scheme to a simulated survey. We estimate that it takes about 2 days for the full SKA to time all the new pulsars just once.

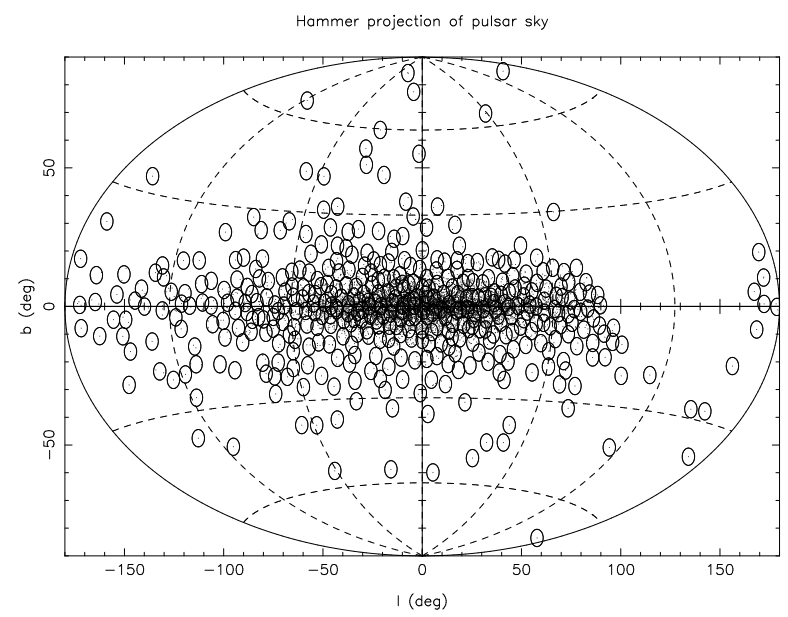

Figure 6: Hammer projection of 15000 detected pulsars from an SKA pulsar survey simulation. The circles indicate all pointings (700) that result from the timing optimization method, assuming a FoV of $20 \mathrm{deg}^{2}$.

\section{References}

Lorimer, D. R., Faulkner, A. J., Lyne, A. G., et al. 2006, MNRAS, 372, 777 